\title{
On a Class of Alternatingly Hyperexpansive Subnormal Weighted Shifts
}

\author{
AMEER ATHAVALE, ABHIJIT RANJEKAR AND V. M. SHOLAPURKAR
}

\begin{abstract}
If $T$ is a weighted shift operator on a Hilbert space with the associated weight sequence $\left\{\alpha_{n}\right\}_{n} \geq 0$ of positive weights, then meaningful insights into the nature of $T$ can be gained by examining the sequence $\left\{\theta_{n}(T)\right\}_{n \geq 0}$ where $\theta_{0}(T)=1$ and $\theta_{n}(T)=\Pi_{k=0}^{n-1} \alpha_{k}^{2}(n \geq 1)$. We characterize those subnormal weighted shifts whose associated $\theta_{n}(T)$ are interpolated by members of a special subclass of the class of absolutely monotone functions on the non-negative real line. The special subclass has such pleasant properties as being closed under differentiation and integration. We also attempt to highlight the operator theoretic significance of such characterizations.
\end{abstract}

\section{INTRODUCTION}

Let $\mathcal{H}$ be a complex infinite-dimensional separable Hilbert space. By $\mathcal{B}(\mathcal{H})$ we will denote the algebra of bounded linear operators on $\mathcal{H}$. If $\left\{e_{n}\right\}_{n \geq 0}$ is an orthonormal basis for $\mathcal{H}$, then a weighted shift operator $T$ on $\mathcal{H}$ with the weight sequence $\left\{\alpha_{n}=\alpha_{n}(T)\right\}_{n \geq 0}$ is defined through the relations $T e_{n}=\alpha_{n} e_{n+1}(n \geq 0)$. We will always assume that $\alpha_{n}>0$ for all $n$, and that $\left\{\alpha_{n}\right\}_{n \geq 0}$ is a bounded sequence so that $T$ is in $\mathcal{B}(\mathcal{H})$. The basic properties of weighted shift operators can be found in [3] and [10]. We will use the notation $T=\Gamma\left(\alpha_{n}\right)$ to indicate a weighted shift with the weight sequence $\left\{\alpha_{n}\right\}_{n \geq 0}$. For a weighted shift $T=\Gamma\left(\alpha_{n}\right)$, the sequence $\left\{\theta_{n}=\theta_{n}(T)\right\}_{n>0}$ is defined by $\theta_{0}=1, \theta_{n}=\prod_{k=0}^{n-1} \alpha_{k}^{2}(n \geq 1)$. Note that $\theta_{n}=\left\|T^{n} e_{0}\right\|^{2}$ and $\alpha_{n}=\sqrt{\theta_{n+1} / \theta_{n}}, n \geq 0$. An operator $T$ in $\mathcal{B}(\mathcal{H})$ is said to be subnormal if there exist a Hilbert space $\mathcal{K}$ containing $\mathcal{H}$ and a normal operator $N$ in $\mathcal{B}(\mathcal{K})$ such that $N \mathcal{H} \subset \mathcal{H}$ and $N_{\mid \mathcal{H}}=T$.

2000 Mathematics Subject Classification. 47B20, 47B37, 47B39; 05A19, 11B73, $11 \mathrm{~B} 83$. 
An operator $\mathrm{T}$ in $\mathcal{B}(\mathcal{H})$ is said to be alternatingly hyperexpansive if $(-1)^{n} \sum_{0 \leq p \leq n}(-1)^{p}\left(\begin{array}{l}n \\ p\end{array}\right) T^{* p} T^{p} \geq 0$ for all $n \geq 1$. The Bergman shift $B=\Gamma\left(\sqrt{\frac{n+1}{n+2}}\right)$ is subnormal, while the Dirichlet shift $T=\Gamma\left(\sqrt{\frac{n+2}{n+1}}\right)$ and the weighted shift $T=\Gamma\left(\frac{n+2}{n+1}\right)$ are alternatingly hyperexpansive; any isometry is subnormal as well as alternatingly hyperexpansive. While there is a copious amount of literature on subnormal operators (refer to [3] for references), some basic facts pertaining to alternatingly hyperexpansive operators have been recorded in [1], [7] and [9].

The symbol $\mathbb{R}_{+}$will denote the set $[0, \infty)$ of non-negative reals, while the symbol $\mathbb{N}$ will stand for the set of non-negative integers. For a real-valued map $\varphi$ on $\mathbb{N}$ we define the (forward) difference operator $\Delta$ as follows: $(\Delta \varphi)(n)=\varphi(n)-\varphi(n+1)$. The operators $\Delta^{n}$ are inductively defined for all $n \geq 0$ through the relations $\Delta^{0} \varphi=$ $\varphi, \Delta^{n} \varphi=\Delta\left(\Delta^{n-1} \varphi\right)(n \geq 1)$. A non-negative map $\varphi$ on $\mathbb{N}$ is said to be absolutely monotone if $\left(\Delta^{k} \varphi\right)(n) \geq 0$ for all $k, n \geq 0$.

A sequence $\left\{\beta_{n}\right\}_{n \geq 0}$ of reals will be referred to as a moment sequence if there exist a positive real number $b$ and a positive Borel measure $\mu$ on $[0, b]$ such that

$$
\beta_{n}=\int_{[0, b]} x^{n} d \mu(x) \quad(n \geq 0) .
$$

It is well known that a weighted shift $T=\Gamma\left(\alpha_{n}\right)$ is subnormal if and only if the sequence $\left\{\theta_{n}(T)\right\}_{n \geq 0}$ is a moment sequence corresponding to a probability measure on $\left[0,\|T\|^{2}\right]$ (refer to [4]).

For $x$ in $\mathbb{R}_{+}$and $k$ in $\mathbb{N}$, let $(x)_{k}$ denote the "falling factorial of $x$ ", that is, $(x)_{0}=1$ and $(x)_{k}=x(x-1) \ldots(x-k+1)$ for $k \geq 1$. Given a sequence $\left\{\beta_{n}\right\}_{n \geq 0}$ of reals, we have by Newton's Interpolation Formula, $\beta_{n}=(1+\triangle)^{n} \beta_{0}$, that is,

$$
\beta_{n}=\sum_{k=0}^{n} a_{k}(n)_{k}
$$

where $a_{k}=\triangle^{k} \beta_{0} / k$ !

It was noted in [9] that a sequence $\left\{\beta_{n}\right\}_{n \geq 0}$ of reals is absolutely monotone if and only if $a_{k} \geq 0$ for all $k$ in (2). Further, as was recorded in [9], a weighted shift $T=\Gamma\left(\alpha_{n}\right)$ is alternatingly hyperexpansive if and only if the sequence $\left\{\theta_{n}(T)\right\}_{n \geq 0}$ is absolutely monotone. 
A continuous non-negative function on $\mathbb{R}_{+}$is said to be absolutely monotone (on $\mathbb{R}_{+}$) if $f$ is infinitely differentiable on $(0, \infty)$ and satisfies $f^{(n)}(s) \geq 0$ at all points $s$ of $(0, \infty)$ and for all $n \geq 0$. It is well known that an absolutely monotone function has a power series representation on $\mathbb{R}_{+}$(refer to [11]). A sequence $\left\{\beta_{n}\right\}_{n \geq 0}$ is said to be interpolated by a function $f$ defined on $\mathbb{R}_{+}$if $f(n)=\beta_{n}$ for all $n$ in $\mathbb{N}$. While it is known that any sequence interpolated by an absolutely monotone function is absolutely monotone (see [11], for example), it was observed in [9] that not every absolutely monotone sequence arises that way.

In the sequel, it will be convenient to use the symbol $\mathrm{AH}$ to refer to the class of alternatingly hyperexpansive operators (on a given Hilbert space), and the symbols $\mathcal{A} M S$ and $\mathcal{A} M F$, respectively to refer to the class of absolutely monotone sequences and the class of absolutely monotone functions on $\mathbb{R}_{+}$, respectively. The intersection of the class AH with the class of subnormals defies an easy description. Indeed, as was shown in Proposition 4.1 of [9], if a subnormal operator $T$ is such that the spectrum of its 'minimal' normal extension is contained in the complement of the open unit disk in the complex plane centered at the origin, then $T$ is alternatingly hyperexpansive; on the other hand there exist alternatingly hyperexpansive subnormal operators such that the spectrum of their minimal normal extension intersects the open unit disk-consider, for example, the weighted shift $T=\Gamma(\sqrt{\cosh (n+1) / \cosh (n)})$ for which $\left\{\theta_{n}(T)=\cosh (n)\right\}_{n \geq 0}$ is a moment sequence with the corresponding measure $\mu$ concentrated at the points $e$ and $1 / e$. In the context of weighted shifts, and in view of our discussion above, an almost tautological answer to the problem under consideration is: A weighted shift $T$ is subnormal as well as in AH if and only if $\left\{\theta_{n}(T)\right\}_{n \geq 0}$ is an absolutely monotone moment sequence. A natural question to raise, then, is: Can one characterize those subnormal weighted shifts $T$ whose associated $\left\{\theta_{n}(T)\right\}_{n \geq 0}$ are interpolated by members of $\mathcal{A} M F$ ? While the question could be of interest in its own right, Theorem 1 below provides a strong motivation for examining such sequences; indeed, the statement of Theorem 1 is the statement of a 'spectral permanence property' of $T$ under the operations of differentiation and integration on the corresponding member of $\mathcal{A M F}$.

As has been pointed out in [2], there is not much literature on absolutely monotone sequences. To the knowledge of the authors, 
there is no known characterization of absolutely monotone sequences interpolated by absolutely monotone functions; to that extent, the question posed above appears difficult to answer. One could, however, hope to obtain some positive results by looking at special subclasses of $\mathcal{A} M F$. In Section 2 we introduce a special subclass $\mathcal{A} M F^{*}$ of $\mathcal{A} M F$, and in Section 3 we provide an explicit characterization of those moment sequences that are interpolated by members of $\mathcal{A} M F^{*}$. The class $\mathcal{A} M F^{*}$ enjoys several pleasant properties; in particular, we establish that $\mathcal{A} M F^{*}$ is closed under the operations of differentiation and integration and allows the action of differentiation to be interpreted as $\log (1+\Delta)$.

We conclude the present section by attempting to highlight the operator theoretic motivation underlying the considerations in Sections 2 and 3 .

If $T=\Gamma\left(\alpha_{n}\right)$ is a weighted shift operator such that $\lim _{n \rightarrow \infty} \alpha_{n}=\alpha$ exists, then it is well known (see [3]) that the assertions (S1), (S2) and (S3) below hold:

(S1) The spectrum $\sigma(T)$ of $T$ equals $\{\lambda:|\lambda| \leq \alpha\}$.

(S2) The essential spectrum $\sigma_{e}(T)$ of $T$ equals $\{\lambda:|\lambda|=\alpha\}$.

(S3) If $|\lambda|<\alpha$, then $T-\lambda I$ is Fredholm with the Fredholm index $\operatorname{ind}(T-\lambda I)$ of $T-\lambda I$ being equal to -1 .

Lemma 1. Suppose $f: \mathbb{R}_{+} \rightarrow \mathbb{R}_{+} \backslash\{0\}$ is such that $f(s)=$ $\int_{(0, b]} x^{s} d \mu(x)$ with $\mu$ a positive Borel measure and $b$ a positive real. Then $\lim _{s \rightarrow \infty} \frac{f(s+1)}{f(s)}$ exists and equals the $\mu$-essential sup norm $\|x\|_{\infty}$ of $x$.

Proof. With $f$ as above, we have $\lim _{n \rightarrow \infty} \frac{f(n+1)}{f(n)}=\|x\|_{\infty}$ (see [8]). Let $[s]$ denote the integral part of $s$ and let

$$
g(s)=\int_{(0, b]}\left(x /\|x\|_{\infty}\right)^{[s]} d \mu(x) \quad(s \geq 0) .
$$

Using $[s] \leq s<[s]+1,[s+n]=[s]+n(n \in \mathbb{N})$, and $x /\|x\|_{\infty} \leq 1$ $([\mu]$-a.e. $)$, we have

$$
\|x\|_{\infty} \frac{g(s+2)}{g(s)} \leq \frac{f(s+1)}{f(s)} \leq\|x\|_{\infty} \frac{g(s)}{g(s+1)}(s \geq 0) .
$$


Let now

$$
g_{1}(s)=\frac{g(s+1)}{g(s)}=\frac{1}{\|x\|_{\infty}} \frac{f([s]+1)}{f([s])}, s \geq 0 .
$$

Clearly, $g_{1}(s)=\frac{1}{\|x\|_{\infty}} \frac{f(n+1)}{f(n)}$ for $n \leq s<n+1$, so that

$$
\lim _{s \rightarrow \infty} g_{1}(s)=\frac{1}{\|x\|_{\infty}} \lim _{n \rightarrow \infty} \frac{f(n+1)}{f(n)}=1 .
$$

Appealing to the inequalities in (3) we arrive at the assertion $\lim _{s \rightarrow \infty} \frac{f(s+1)}{f(s)}=\|x\|_{\infty}$.

Theorem 1. Let $T=\Gamma\left(\alpha_{n}\right)$ be a weighted shift operator which is both subnormal and alternatingly hyperexpansive, and such that the sequence $\left\{\theta_{n}(T)\right\}_{n>0}$ is interpolated by an absolutely monotone function $f(x)=\sum_{j \geq 0} \bar{b}_{j} x^{j}(x \geq 0)$ satisfying $f(0)=1$ and $f(s)=$ $\int_{(0, b]} x^{s} d \mu(x)(s>0)$ with $\mu$ a positive Borel measure and $b$ a positive real. Let $T^{d}$ and $T^{i}$ be the weighted shift operators with $\theta_{n}\left(T^{d}\right)=$ $\frac{1+f^{\prime}(n)}{1+f^{\prime}(0)}$ and $\theta_{n}\left(T^{i}\right)=F(n)$ where $F(x)=1+\sum_{j \geq 0} \frac{b_{j}}{j+1} x^{j+1}(x \geq 0)$. If $\alpha$ is the $\mu$-essential sup norm $\|x\|_{\infty}$ of $x$, then all of $T, T^{d}$ and $T^{i}$ satisfy (S1), (S2) and (S3).

Proof. With $T$ as above we have, from the well-known property of subnormals, that $\lim _{n \rightarrow \infty} \alpha_{n}=\lim _{n \rightarrow \infty} \frac{f(n+1)}{f(n)}$ exists; from our observations in Lemma 1 it is clear that this limit equals $\alpha=\|x\|_{\infty}$, and that $\lim _{s \rightarrow \infty} \frac{f(s+1)}{f(s)}=\alpha$. While the desired conclusion for $T$ is now obvious, that for $T^{i}$ follows from noting that $F^{\prime}(s)=f(s)$ and applying L'Hopital's Rule. Since $f^{\prime \prime}(s)=\int_{(0, b]} x^{s}(\log x)^{2} d \mu(x)$, we have by Lemma 1 again that $\beta=\lim _{s \rightarrow \infty} \frac{f^{\prime \prime}(s+1)}{f^{\prime \prime}(s)}$ exists. In view of L'Hopital's Rule, $\lim _{s \rightarrow \infty} \frac{1+f^{\prime}(s+1)}{1+f^{\prime}(s)}$ and $\lim _{s \rightarrow \infty} \frac{f^{\prime}(s+1)}{f^{\prime}(s)}$ must exist and equal $\beta$. Yet another application of L'Hopital's Rule shows that $\beta$ equals $\alpha$ and the desired conclusion regarding $T^{d}$ follows.

The weighted shift operator $T=\Gamma(\sqrt{\cosh (n+1) / \cosh (n)})$ satisfies the hypotheses of Theorem 1 so that $T$ and $T^{d}=T^{i}=$ $\Gamma(\sqrt{(1+\sinh (n+1)) /(1+\sinh (n))})$ satisfy (S1), (S2) and (S3); it 
should be noted that $T^{d}=T^{i}=\Gamma(\sqrt{(1+\sinh (n+1)) /(1+\sinh (n))})$ is not subnormal.

\section{The Subclass $\mathcal{A} M F^{*}$ of $\mathcal{A} M F$}

In the sequel we require a number of combinatorial properties of Stirling numbers $S_{1}(n, k)$ of the first kind and Stirling numbers $S_{2}(n, k)$ of the second kind $(n, k \in \mathbb{N}) ; S_{1}(n, k)$ and $S_{2}(n, k)$ may formally be defined through relations (D1) and(D2) below as considered valid for any real number $x$. For the basic information on Stirling numbers, the reader is referred to [5]. Some elementary facts regarding Stirling numbers are: $S_{1}(0,0)=S_{2}(0,0)=1 ; S_{1}(n, 0)=S_{2}(n, 0)=0$ $(n \geq 1) ; S_{1}(n, 1)=(n-1) !(n \geq 1) ; S_{2}(n, 1)=1(n \geq 1) ; S_{2}(n, 2)=$ $2^{n-1}-1(n \geq 1) ; S_{1}(n, n)=S_{2}(n, n)=1(n \geq 0) ; S_{1}(n, k)=$ $S_{2}(n, k)=0(n<k)$.

Further, we require recurrence relations (R1), (R2) (with $n, k$ in $\mathbb{N} \backslash\{0\}$ ), inversion formulas (I1), (I2), equalities (E1), (E2), (E3), and "generating function formulas" (G1), (G2) (with $n, k, m$ in $\mathbb{N}$ ) as given below and for which the reader is referred to Chapters 6 and 7 of [5].

(D1) $(x)_{n}=\sum_{k=0}^{n} S_{1}(n, k)(-1)^{n-k} x^{k}$

(D2) $x^{n}=\sum_{k=0}^{n} S_{2}(n, k)(x)_{k}$

(R1) $S_{1}(n, k)=(n-1) S_{1}(n-1, k)+S_{1}(n-1, k-1)$

(R2) $S_{2}(n, k)=k S_{2}(n-1, k)+S_{2}(n-1, k-1)$

(I1) $\sum_{k \geq 0} S_{1}(n, k) S_{2}(k, m)(-1)^{n-k}=\delta_{n, m}$

(I2) $\sum_{k \geq 0} S_{2}(n, k) S_{1}(k, m)(-1)^{n-k}=\delta_{n, m}$

(E1) $S_{2}(n+1, m+1)=\sum_{k \geq 0}\left(\begin{array}{l}n \\ k\end{array}\right) S_{2}(k, m)$

(E2) $m ! S_{2}(n, m)=\sum_{k \geq 0}\left(\begin{array}{c}m \\ k\end{array}\right) k^{n}(-1)^{(m-k)}$

(E3) $\left(\begin{array}{c}n \\ m\end{array}\right)=\sum_{k \geq 0} S_{2}(n+1, k+1) S_{1}(k, m)(-1)^{(m-k)}$

(G1) $\left(\log \frac{1}{1-x}\right)^{m}=m ! \sum_{k \geq 0} S_{1}(k, m) \frac{x^{k}}{k !} \quad(-1<x<1)$

(G2) $\frac{1}{(1-x)^{w}}=\sum_{k \geq 0} \sum_{r \geq 0} S_{1}(k, r) w^{r} \frac{x^{k}}{k !} \quad\left(-1<x<1, w \in \mathbb{R}_{+}\right)$.

Given a sequence $\left\{\gamma_{n}=\sum_{k=0}^{n} a_{k}(n)_{k}\right\}_{n \geq 0}$ of reals, we define, for $r \geq 0$

$$
b_{r}=\sum_{k \geq 0}(-1)^{k-r} a_{k} S_{1}(k, r) .
$$


We use $\mathcal{A} M S^{*}$ to denote the set of those sequences $\left\{\gamma_{n}\right\}_{n \geq 0}$ in $\mathcal{A} M S$ that satisfy conditions (A) and (B) below:

(A) $\sum_{k \geq 0} \sum_{r \geq 0} a_{k} S_{1}(k, r) n^{r}<\infty$ for each $n \in \mathbb{N}$ (here $0^{0}$ is interpreted as the number 1$)$;

(B) $\quad b_{r} \geq 0$.

Further, we use $\mathcal{A} M F^{*}$ to denote the set of those functions $f(x)=$ $\sum_{r \geq 0} c_{r} x^{r}$ in $\mathcal{A} M F$ for which

(C) $\sum_{j \geq 0} \sum_{k \geq 0} \sum_{r \geq 0} c_{j} S_{2}(j, k) S_{1}(k, r) n^{r}<\infty$ for each $n \in \mathbb{N}$.

Theorem 2. Every sequence $\left\{\gamma_{n}\right\}_{n \geq 0}$ in $\mathcal{A} M S^{*}$ is interpolated by a member of $\mathcal{A M F ^ { * }}$. Conversely, any sequence $\left\{\gamma_{n}\right\}_{n \geq 0}$ that is interpolated by a member of $\mathcal{A} M F^{*}$ is a member of $\mathcal{A M S}$.

Proof. If $\left\{\gamma_{n}\right\}_{n \geq 0}$ is in $\mathcal{A} M S^{*}$, then it follows from condition (A) that $\sum_{r \geq 0} b_{r} n^{r}<\infty$ for all $n \geq 0$. Consider the function $f(x)=$ $\sum_{r \geq 0} b_{r} x^{r}$ on $\mathbb{R}_{+}$. Clearly $f$ is a convergent power series on $\mathbb{R}_{+}$ and condition (B) shows that $f$ is an absolutely monotone function. Further, $f(n)=\sum_{r \geq 0} b_{r} n^{r}=\sum_{r \geq 0}\left[\sum_{k \geq 0}(-1)^{k-r} a_{k} S_{1}(k, r)\right] n^{r}=$ $\sum_{k \geq 0} a_{k}\left[\sum_{r \geq 0}(-1)^{k-r} S_{1}(k, r) n^{r}\right]=\sum_{k \geq 0} a_{k}(n)_{k}=\gamma_{n}$ (the interchange of summations is justified by condition (A)). Hence $f$ is an absolutely monotone function that interpolates the sequence $\left\{\gamma_{n}\right\}_{n \geq 0}$. Further, $\gamma_{n}=f(n)=\sum_{r \geq 0} b_{r} n^{r}=\sum_{r \geq 0} b_{r}\left[\sum_{k \geq 0} S_{2}(r, k)(n)_{k}\right]=$ $\sum_{k \geq 0}\left[\sum_{r \geq 0} S_{2}(r, k) b_{r}\right](n)_{k}$ so that $a_{k}=\sum_{r \geq 0} S_{2}(r, k) b_{r}$. Also,

$$
\begin{aligned}
& \sum_{j \geq 0} \sum_{k \geq 0} \sum_{r \geq 0} b_{j} S_{2}(j, k) S_{1}(k, r) n^{r} \\
&=\sum_{r \geq 0} \sum_{k \geq 0}\left[\sum_{j \geq 0} b_{j} S_{2}(j, k)\right] S_{1}(k, r) n^{r} \\
&=\sum_{r \geq 0} \sum_{k \geq 0} a_{k} S_{1}(k, r) n^{r}<\infty
\end{aligned}
$$

by condition (A). Hence the function $f$ interpolating the sequence $\left\{\gamma_{n}\right\}_{n \geq 0}$ satisfies condition (C) and is a member of $\mathcal{A} M F^{*}$.

Conversely, for $f=\sum_{k \geq 0} c_{k} x^{k}$ in $\mathcal{A} M F^{*}$, consider the absolutely monotone sequence $\left\{\gamma_{n}=f(n)=\sum_{k=0}^{n} a_{k}(n)_{k}\right\}_{n \geq 0}$ interpolated by $f$. From our observations above it follows that $a_{k}=$ 
$\sum_{r \geq 0} S_{2}(r, k) c_{r}$. Now,

$$
\begin{aligned}
b_{j} & =\sum_{k \geq 0}(-1)^{k-j} a_{k} S_{1}(k, j) \\
& =\sum_{k \geq 0}\left[\sum_{r \geq 0} S_{2}(r, k) c_{r}\right](-1)^{k-j} S_{1}(k, j) .
\end{aligned}
$$

Since the function $f$ is a member of $\mathcal{A} M F^{*}$, the series representing $b_{j}$ is absolutely convergent so that we have

$$
\begin{aligned}
b_{j} & =\sum_{r \geq 0}\left[\sum_{k \geq 0}(-1)^{k-r} S_{2}(r, k) S_{1}(k, j)\right](-1)^{r-j} c_{r} \\
& =\sum_{r \geq 0} \delta(r, j)(-1)^{r-j} c_{r}=c_{j} .
\end{aligned}
$$

Thus the sequence $\left\{\gamma_{n}=f(n)\right\}_{n \geq 0}$ satisfies condition (B). Further,

$$
\begin{aligned}
\sum_{k \geq 0} \sum_{r \geq 0} a_{k} & S_{1}(k, r) n^{r} \\
= & \sum_{k \geq 0} \sum_{r \geq 0} \sum_{p \geq 0} S_{2}(p, k) c_{p} S_{1}(k, r) n^{r}<\infty,
\end{aligned}
$$

since the function $f$ is a member of $\mathcal{A} M F^{*}$. Thus the sequence $\left\{\gamma_{n}=f(n)\right\}_{n \geq 0}$ satisfies condition (A) as well.

Examples 1. (i) Any sequence $\left\{\beta_{n}\right\}_{n \geq 0}$ for which $\beta_{n}=p(n)$, where $p(n)$ is a polynomial with non-negative coefficients, is a member of $\mathcal{A} M S^{*}$. If, moreover, $p(0)=1$, then $p(n)$ may be looked upon as $\theta_{n}(T)$ corresponding to a weighted shift $T$ that is an alternatingly hyperexpansive $d$-isometry (refer to [9]). Besides the trivial case of the constant polynomial $p(z)=1$, the weighted shifts so obtained are necessarily non-subnormal.

(ii) For the sequence $\left\{\beta_{n}=(1+\delta)^{n}\right\}_{n \geq 0}$, with $0 \leq \delta<1$, we have $a_{k}=\delta^{k} / k$ !. Since $0 \leq \delta<1$, we have, in view of identity (G2), $\sum_{k \geq 0} \sum_{r \geq 0} a_{k} S_{1}(k, r) n^{r}=\sum_{k \geq 0} \sum_{r \geq 0} S_{1}(k, r) \frac{\delta^{k}}{k !} n^{r}=\frac{1}{(1-\delta)^{n}}<\infty$ for all $n \geq 0$. Hence $\left\{\beta_{n}\right\}_{n \geq 0}$ satisfies condition (A).

Further, $b_{r}=\sum_{k \geq 0}(-1)^{k-r} S_{1}(k, r) a_{k}=\sum_{k \geq 0}(-1)^{k-r} S_{1}(k, r) \frac{\delta^{k}}{k !}$. Since $0 \leq \delta<1$, we have $b_{r}=\frac{1}{r !}[\log (1+\delta)]^{r}(r \geq 0)$, in view of identity (G1). Thus $\left\{\beta_{n}\right\}_{n \geq 0}$ satisfies condition (B) as well. It is seen that $\left\{\beta_{n}\right\}_{n \geq 0}$ is interpolated by the absolutely monotone function 
$f(x)=\sum_{r \geq 0} b_{r} x^{r}=\sum_{r \geq 0} \frac{1}{r !}[\log (1+\delta)]^{r} x^{r}=e^{x \log (1+\delta)}=(1+\delta)^{x}$. We will return to this example following Theorem 6 below.

Theorems 3 and 5 below show that the class $\mathcal{A} M F^{*}$ is closed under the operations of differentiation and integration, while Theorem 4 below deciphers the action of differentiation on that class.

Theorem 3. If $f$ belongs to $\mathcal{A} M F^{*}$ then the derivatives $f^{(k)}$ belong to $\mathcal{A} M F^{*}$ for all $k \geq 0$.

Proof. If $f(x)=\sum_{j \geq 0} b_{j} x^{j}$ then $f^{\prime}(x)=\sum_{j \geq 0} c_{j} x^{j}$, where $c_{j}=$ $(j+1) b_{j+1}$. In view of identity (E1) we have

$$
(j+1) S_{2}(j, k) \leq S_{2}(j+2, k+1) .
$$

Thus

$$
\begin{aligned}
\sum_{j \geq 0} \sum_{k \geq 0} \sum_{r \geq 0} & (j+1) b_{j+1} S_{2}(j, k) S_{1}(k, r) n^{r} \\
\leq & \sum_{j \geq 0} \sum_{k \geq 0} \sum_{r \geq 0} b_{j+1} S_{2}(j+2, k+1) S_{1}(k, r) n^{r} \\
& =P_{1}+P_{2}+P_{3},
\end{aligned}
$$

where

$$
\begin{aligned}
& P_{1}=\sum_{j \geq 0} \sum_{k \geq 0} \sum_{r \geq 0} b_{j+1} S_{2}(j+1, k+1) k S_{1}(k, r) n^{r}, \\
& P_{2}=\sum_{j \geq 0} \sum_{k \geq 0} \sum_{r \geq 0} b_{j+1} S_{2}(j+1, k+1) S_{1}(k, r) n^{r}
\end{aligned}
$$

and

$$
P_{3}=\sum_{j \geq 0} \sum_{k \geq 0} \sum_{r \geq 0} b_{j+1} S_{2}(j+1, k) S_{1}(k, r) n^{r} .
$$

(Here we used the recurrence relation (R2) for $S_{2}(j+2, k+1)$.) Since $f$ is a member of $\mathcal{A} M F^{*}$, we have $P_{3}<\infty$. The finiteness of $P_{1}$ and that of $P_{2}$ follow by noting that $k S_{1}(k, r) \leq S_{1}(k+1, r)$ and $S_{1}(k, r) \leq S_{1}(k+1, r+1)$ and using condition (C) for the function $f$. Thus $f^{\prime}$ satisfies condition (C) and hence belongs to $\mathcal{A} M F^{*}$.

The difference operator $\triangle$ can be interpreted to act on any function $f$ defined on $\mathbb{R}_{+}$through the relation $\triangle f(x)=f(x+1)-f(x)$. Interpreted thus, $\triangle$ acts on any real analytic function $f$ as $e^{D}-1$, 
where $D$ denotes differentiation (refer to [5], Chapter 9). It is further known (refer to [6], Chapter 5) that $D$ acts on polynomials as $\log (1+\triangle)$ (see Definition 1 below). In Theorem 4 below, we establish that $D$ effectively acts as $\log (1+\triangle)$ on any member of the class $\mathcal{A} M F^{*}$.

Lemma 2. (a) If $\left\{\gamma_{n}=\sum_{k=0}^{n} a_{k}(n)_{k}\right\}_{n \geq 0}$ is in $\mathcal{A} M S^{*}$, then $\sum_{k \geq 0} \frac{\triangle^{k} \gamma_{d}}{k !} S_{1}(k, r)<\infty$ for all non-negative integers $d$ and $r$.

(b) If $f$ is in $\mathcal{A} M F^{*}$, then $\sum_{p \geq 0} \sum_{k \geq 0} \frac{f^{p}(d)}{p !} S_{2}(p, k) S_{1}(k, r)<\infty$ for all non-negative integers $d$ and $r$.

Proof. (a) For the sequence $\left\{\tilde{\gamma}_{n}=\gamma_{n+1}=\sum_{k=0}^{n} \tilde{a}_{k}(n)_{k}\right\}$, we have $\tilde{a_{k}}=\triangle^{k} \tilde{\gamma_{0}} / k !=\triangle^{k} \gamma_{1} / k !=\triangle^{k}(1+\triangle) \gamma_{0} / k !=\triangle^{k} \gamma_{0} / k !+$ $\triangle^{k+1} \gamma_{0} / k !=a_{k}+(k+1) a_{k+1}$. Consider

$$
\begin{aligned}
\sum_{k \geq 0} \sum_{r \geq 0} \tilde{a_{k}} S_{1}(k, r) n^{r} & =\sum_{k \geq 0} \sum_{r \geq 0}\left[a_{k}+(k+1) a_{k+1}\right] S_{1}(k, r) n^{r} \\
& =P_{1}+P_{2}+P_{3},
\end{aligned}
$$

$P_{1}=\sum_{k>0} \sum_{r>0} a_{k} S_{1}(k, r) n^{r}, P_{2}=\sum_{k>0} \sum_{r>0} k a_{k+1} S_{1}(k, r) n^{r}$ and $P_{3}=\sum_{k \geq 0} \sum_{r \geq 0} a_{k+1} S_{1}(k, r) n^{r}$. The finiteness of $P_{1}$ follows from condition (A). Using the recurrence relation (R1), one has $k S_{1}(k, r) \leq S_{1}(k+1, r)$, leading to the finiteness of $P_{2}$. Further, using the same recurrence relation, one has $S_{1}(k, r) \leq S_{1}(k+1, r+1)$, leading to the finiteness of $P_{3}$. Thus $\left\{\tilde{\gamma}_{n}\right\}$ satisfies condition (A). Since $\left\{\gamma_{n}\right\}_{n \geq 0}$ is in $\mathcal{A} M S^{*}$, there exists a function $f(x)=\sum_{p \geq 0} b_{p} x^{p}$ in $\mathcal{A} M F^{*}$ such that $\gamma_{n}=f(n)$ for $n \in \mathbb{N}$. Hence,

$$
\begin{aligned}
\triangle^{k} \tilde{\gamma_{0}}=\triangle^{k} \gamma_{1}=\triangle^{k} f(1) & =\sum_{r=0}^{k}\left(\begin{array}{l}
k \\
r
\end{array}\right)(-1)^{k-r} f(1+r) \\
& =\sum_{r=0}^{k}\left(\begin{array}{l}
k \\
r
\end{array}\right)(-1)^{k-r}\left[\sum_{p \geq 0} b_{p}(1+r)^{p}\right] \\
& =\sum_{p \geq 0}\left[\sum_{r=0}^{k}\left(\begin{array}{l}
k \\
r
\end{array}\right)(-1)^{k-r}(1+r)^{p}\right] b_{p} \\
& =\sum_{p \geq 0} d(k, p) b_{p} .
\end{aligned}
$$


Now, by (E2),

$$
\begin{aligned}
d(k, p) & =\sum_{r=0}^{k}\left(\begin{array}{l}
k \\
r
\end{array}\right)(-1)^{k-r}(1+r)^{p} \\
& =\sum_{r=0}^{k} \frac{1+r}{k+1}\left(\begin{array}{c}
k+1 \\
r+1
\end{array}\right)(-1)^{k-r}(1+r)^{p} \\
& =\frac{1}{k+1} \sum_{r=0}^{k}\left(\begin{array}{l}
k+1 \\
r+1
\end{array}\right)(-1)^{k-r}(1+r)^{p+1} \\
& =\frac{1}{k+1}(k+1) ! S_{2}(p+1, k+1) \\
& =k ! S_{2}(p+1, k+1) .
\end{aligned}
$$

Hence, $\tilde{a_{k}}=\triangle^{k} \tilde{\gamma_{0}} / k !=\sum_{p \geq 0} d(k, p) b_{p} / k !=\sum_{p \geq 0} S_{2}(p+1, k+1) b_{p}$.

Thus,

$$
\begin{aligned}
\tilde{b_{r}} & \equiv \sum_{k \geq 0} S_{1}(k, r)(-1)^{k-r} \tilde{a_{k}} \\
& =\sum_{k \geq 0} S_{1}(k, r)(-1)^{k-r}\left[\sum_{p \geq 0} S_{2}(p+1, k+1) b_{p}\right] \\
& =\sum_{p \geq 0}\left[\sum_{k \geq 0} S_{2}(p+1, k+1) S_{1}(k, r)(-1)^{k-r}\right] b_{p} \\
& =\sum_{p \geq 0}\left(\begin{array}{l}
p \\
r
\end{array}\right) b_{p},
\end{aligned}
$$

where in the last step we used identity (E3). Since $\sum_{p \geq 0}\left(\begin{array}{l}p \\ r\end{array}\right) b_{p}=$ $f^{r}(1) / r$ !, we have $0 \leq \tilde{b_{r}}<\infty$. (The justification for the interchange of summations can be provided as follows:

$$
\begin{aligned}
\sum_{p \geq 0} \sum_{k \geq 0} & S_{2}(p+1, k+1) S_{1}(k, r) b_{p} \\
& =\sum_{p \geq 0} \sum_{k \geq 0}\left[S_{2}(p, k)+(k+1) S_{2}(p, k+1)\right] S_{1}(k, r) b_{p} \\
& =Q_{1}+Q_{2}+Q_{3},
\end{aligned}
$$


where

$$
\begin{aligned}
Q_{1} & =\sum_{p \geq 0} \sum_{k \geq 0} S_{2}(p, k) S_{1}(k, r) b_{p}, \\
Q_{2} & =\sum_{p \geq 0} \sum_{k \geq 0} S_{2}(p, k+1) k S_{1}(k, r) b_{p}, \\
Q_{3} & =\sum_{p \geq 0} \sum_{k \geq 0} S_{2}(p, k+1) S_{1}(k, r) b_{p} .
\end{aligned}
$$

Since $f$ is in $\mathcal{A} M F^{*}$, using condition (C) for $n=1$, the finiteness of $Q_{1}$ follows. The finiteness of $Q_{2}$ and that of $Q_{3}$ follow by appealing to the recurrence relations (R1) and (R2) again.) Thus $\left\{\tilde{\gamma}_{n}\right\}$ satisfies condition (B) as well. Hence $\left\{\tilde{\gamma}_{n}\right\}$ is a member of $\mathcal{A} M S^{*}$. It follows that the sequence $\left\{\gamma_{n+d}\right\}_{n \geq 0}$ is in $\mathcal{A} M S^{*}$ for every $d \geq 0$. In particular, $\sum_{k \geq 0} \sum_{r \geq 0} \frac{\triangle^{k} \gamma_{d}}{k !} S_{1}(k, r) n^{r}<\infty$ for every $d \geq 0$. Putting $n=1$, the desired conclusion follows.

(b) If the sequence $\left\{\gamma_{n}\right\}_{n \geq 0}$ belongs to $\mathcal{A} M S^{*}$, then it follows from the proof of Theorem 1 that the function $f(x)=\sum_{r \geq 0} b_{r} x^{r}$ belongs to $\mathcal{A} M F^{*}$. By part (a) above, the sequence $\left\{\left\{\tilde{\gamma}_{n}\right\}\right.$ belongs to $\mathcal{A} M S^{*}$ and hence the function $\tilde{f}(x)=\sum_{r \geq 0} \tilde{b}_{r} x^{r}$ is a member of $\mathcal{A} M F^{*}$. As shown in the proof of part (a) above, we have $\tilde{b}_{r}=f^{r}(1) / r !$; thus $\tilde{f}(x)=\sum_{r>0} f^{r}(1) / r ! x^{r}=f(x+1)$. This shows that $g_{1}(x)=f(x+1)$ is a member of $\mathcal{A} M F^{*}$, and so is $g_{d}(x)=f(x+d)$ for every integer $d \geq 0$. Thus $g_{d}$ satisfies condition (C), that is, $\sum_{p \geq 0} \sum_{k \geq 0} \sum_{r \geq 0} \frac{f^{p}(d)}{p !} S_{2}(p, k) S_{1}(k, r) n^{r}<\infty$ for every $n \geq 0$. Putting $n=1$, the desired conclusion follows.

For $m \in \mathbb{N}$, let $\mathcal{A} M S^{(m)}$ denote the class of absolutely monotone sequences $\left\{\gamma_{n}\right\}_{n \geq 0}$ for which $\sum_{p \geq m} m !(-1)^{p-m} S_{1}(p, m) \frac{\Delta^{p} \gamma_{n}}{p !}$ are finite real numbers for all $n \geq 0$.

Definition 1. Given a sequence $\left\{\gamma_{n}\right\}_{n \geq 0}$ in $\mathcal{A} M S^{(m)}$, define

$$
[\log (1+\triangle)]^{m} \gamma_{n}=\sum_{p \geq m} m !(-1)^{p-m} S_{1}(p, m) \frac{\triangle^{p} \gamma_{n}}{p !} .
$$

(In particular, for $m=1$,

$$
\left.\log (1+\triangle) \gamma_{n}=\sum_{k \geq 1} \frac{(-1)^{k+1}}{k} \triangle^{k} \gamma_{n}=\sum_{k \geq 1}(-1)^{k-1} S_{1}(k, 1) \frac{\triangle^{k} \gamma_{n}}{k !} .\right)
$$


Theorem 4. The inclusion $\mathcal{A} M S^{*} \subset \mathcal{A} M S^{(m)}$ holds for every positive integer $m$; moreover, the following commutative diagram holds with $D$ denoting differentiation:

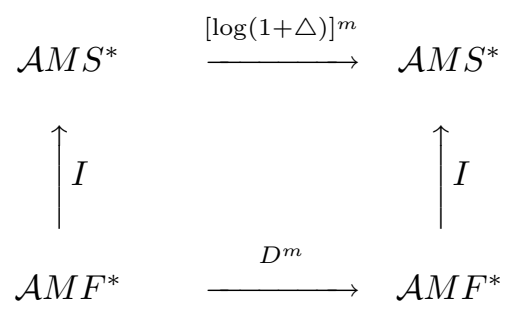

Proof. Suppose a sequence $\left\{\gamma_{n}\right\}_{n \geq 0}$ is interpolated by $f$ in $\mathcal{A} M F^{*}$; then $\left\{\gamma_{n}\right\}_{n \geq 0}$ is a member of $\mathcal{A} M S^{*}$ by Theorem 1 , and by part (a) of Lemma 2 we have that $\sum_{p=0}^{\infty} \frac{\triangle^{p} \gamma_{n}}{p !} S_{1}(p, m)<\infty$ for every $n \geq 0$ and $m \geq 0$. Hence $[\log (1+\triangle)]^{m} \gamma_{n}$ is a finite real number for every $m \geq 0$ and $n \geq 0$. Since $\gamma_{n}=f(n)$ for all $n \geq 0$, we have

$$
\begin{aligned}
\triangle^{p} \gamma_{n}=\triangle^{p} f(n) & =(-1)^{p} \sum_{r=0}^{p}\left(\begin{array}{l}
p \\
r
\end{array}\right)(-1)^{r} f(n+r) \\
& =(-1)^{p} \sum_{r=0}^{p}\left(\begin{array}{l}
p \\
r
\end{array}\right)(-1)^{r}\left[\sum_{k=0}^{\infty} r^{k} f^{(k)}(n) / k !\right] \\
& =\sum_{k=0}^{\infty}\left[\sum_{r=0}^{p}\left(\begin{array}{l}
p \\
r
\end{array}\right)(-1)^{p-r} r^{k}\right] f^{(k)}(n) / k ! \\
& =\sum_{k=0}^{\infty} p ! S_{2}(k, p) f^{(k)}(n) / k !,
\end{aligned}
$$

where in the last step we used identity (E2). Also,

$$
\begin{aligned}
{[\log (1+\triangle)]^{m} \gamma_{n} } & =\sum_{p=0}^{\infty} m !(-1)^{p-m} S_{1}(p, m) \frac{\triangle^{p} \gamma_{n}}{p !} \\
& =\sum_{p=0}^{\infty} m !(-1)^{p-m} S_{1}(p, m)\left[\sum_{k=0}^{\infty} S_{2}(k, p) f^{(k)}(n) / k !\right]
\end{aligned}
$$




$$
\begin{aligned}
& =m ! \sum_{k=0}^{\infty}\left[\sum_{p=0}^{\infty} S_{2}(k, p) S_{1}(p, m)(-1)^{k-p}\right](-1)^{m-k} f^{(k)}(n) / k ! \\
& =m ! \sum_{k=0}^{\infty} \delta_{k, m}(-1)^{m-k} f^{(k)}(n) / k ! \\
& =m ! f^{(m)}(n) / m !=f^{(m)}(n) .
\end{aligned}
$$

(The interchange of summations above is justified by part (b) of Lemma 2.)

Theorem 5. If $f(x)=\sum_{j \geq 0} b_{j} x^{j}$ belongs to $\mathcal{A} M F^{*}$, then the function $F(x)=1+\sum_{j \geq 0} \frac{b_{j}}{j+1} x^{j+1}$ (obtained by integrating the series for $f(x)$ term by term) belongs to $\mathcal{A M F}$.

Proof. If $F(x)=\sum_{j \geq 0} \tilde{b_{j}} x^{j}$ then $\tilde{b_{0}}=1, \tilde{b_{j}}=\frac{b_{j-1}}{j}(j \geq 1)$. Using the recurrence relation (R2) we have

$$
\sum_{j \geq 0} \sum_{k \geq 0} \sum_{r \geq 0} \tilde{b_{j}} S_{2}(j, k) S_{1}(k, r) n^{r}=1+P_{1}+P_{2},
$$

where $P_{1}=\sum_{j \geq 1} \sum_{k \geq 0} \sum_{r \geq 0} \frac{b_{j-1}}{j} k S_{2}(j-1, k) S_{1}(k, r) n^{r}$ and $P_{2}=$ $\sum_{j \geq 1} \sum_{k \geq 0} \sum_{r \geq 0} \frac{b_{j-1}}{j} S_{2}(j-1, k-1) S_{1}(k, r) n^{r}$. For $k>j$ one has $S_{2}(j, k)=0$ so that in $P_{1}$ one may only consider the terms for which $k \leq j$. Thus

$$
P_{1} \leq \sum_{j \geq 1} \sum_{k \geq 0} \sum_{r \geq 0} b_{j-1} S_{2}(j-1, k) S_{1}(k, r) n^{r} .
$$

Further, it follows from the recurrence relation (R1) that $S_{1}(k, r) \leq$ $(k-1) S_{1}(k-1, r-1)$. Thus

$$
P_{2} \leq \sum_{j \geq 1} \sum_{k \geq 0} \sum_{r \geq 0} b_{j-1} S_{2}(j-1, k-1) S_{1}(k-1, r-1) n^{r} .
$$

The finiteness of $P_{1}$ and that of $P_{2}$ follow by using condition (C) for the function $f$. Thus $F$ satisfies condition $(\mathrm{C})$ and hence belongs to $\mathcal{A M F ^ { * }}$.

\section{Moment Sequences in $\mathcal{A} M S^{*}$}

Theorem 6 below provides necessary and sufficient conditions for an absolutely monotone moment sequence to fall in $\mathcal{A} M S^{*}$. 
Theorem 6. Let $\left\{\beta_{n}=\sum_{k>0} a_{k}(n)_{k}\right\}_{n \geq 0}$ be an absolutely monotone sequence as well as a moment sequence with the representation $\beta_{n}=\int_{[0, b]} x^{n} d \mu(x) \quad(n \geq 0)$ (where $\mu$ is a finite positive Borel measure on $[0, b], b>0)$. Then $\left\{\beta_{n}\right\}_{n \geq 0}$ is in $\mathcal{A} M S^{*}$ if and only the measure $\mu$ is supported on the open interval $(0,2)$ and satisfies conditions $\left(\mathrm{A}^{*}\right)$ and $\left(\mathrm{B}^{*}\right)$ below:

$\left(\mathrm{A}^{*}\right) \int_{[1,2)} \frac{1}{(2-x)^{n}} d \mu(x)<\infty$ for every positive integer $n$.

$\left(\mathrm{B}^{*}\right) \int_{(0,2)}(\log x)^{r} d \mu(x) \geq 0$ for every odd positive integer $r$.

Proof. For any sequence $\left\{\beta_{n}\right\}_{n \geq 0}$ as in the hypotheses of the theorem and with $\mu$ supported on $(0,2)$ in particular, one may write $a_{k}=A_{k}+(-1)^{k} B_{k}$, where $A_{k}=\int_{[1,2)} \frac{(x-1)^{k}}{k !} d \mu(x)$ for $k \geq 0$ and $B_{k}=\int_{(0,1)} \frac{(1-x)^{k}}{k !} d \mu(x)$ for $k \geq 0$. Since $a_{k} \geq 0$ for all $k \geq 0$, one has $A_{2 k-1} \geq B_{2 k-1}$ for all $k \geq 1$. Further, $2 k A_{2 k} \leq A_{2 k-1}$ for $k \geq 1$ and $2 k B_{2 k} \leq B_{2 k-1}$ for $k \geq 1$.

Suppose now the sequence $\left\{\beta_{n}\right\}_{n \geq 0}$ belongs to $\mathcal{A} M S^{*}$. We first observe that the measure $\mu$ must be supported on $(0,2)$. Indeed, if $\mu\{[2, b]\}>0$, then it is easy to see that the series $\sum_{k \geq 0} a_{2 k} S_{1}(2 k, 1)$ is divergent; similarly $\mu(\{0\})>0$ leads to the divergence of the same series. Next, $\left\{\beta_{n}\right\}_{n \geq 0}$ satisfies condition (A) so that

$$
\sum_{k \geq 0} \sum_{r \geq 0} a_{k} S_{1}(k, r) n^{r}<\infty
$$

for all $n \geq 0$. Since $a_{2 k} \geq A_{2 k}$ for all $k \geq 0$, one has

$$
\sum_{k \geq 0} \sum_{r \geq 0} A_{2 k} S_{1}(2 k, r) n^{r}<\infty
$$

for all $n \geq 0$. Using the fact $(2 k+1) A_{2 k+1} \leq A_{2 k}$ for $k \geq 0$ and the recurrence relation (R1), one also has $A_{2 k+1} S_{1}(2 k+1, r) n^{r} \leq$ $A_{2 k} S_{1}(2 k, r) n^{r}+n A_{2 k} S_{1}(2 k, r-1) n^{r-1}$ for all $k \geq 0, n \geq 1$. This shows that $\sum_{k \geq 0} \sum_{r \geq 0} A_{2 k+1} S_{1}(2 k+1, r) n^{r}<\infty$ for all $n \geq 0$. Thus $\sum_{k \geq 0} \sum_{r \geq 0} A_{k} \bar{S}_{1}(k, r) n^{r}<\infty$ for all $n \geq 0$. It then follows from

$$
\sum_{k \geq 0} \sum_{r \geq 0} A_{k} S_{1}(k, r) n^{r}=\sum_{k \geq 0} \sum_{r \geq 0}\left[\int_{[1,2)} \frac{(x-1)^{k}}{k !} d \mu(x)\right] S_{1}(k, r) n^{r}
$$




$$
\begin{aligned}
& =\int_{[1,2)}\left[\sum_{k \geq 0} \sum_{r \geq 0} \frac{(x-1)^{k}}{k !} S_{1}(k, r) n^{r}\right] d \mu(x) \\
& =\int_{[1,2)} \frac{1}{(2-x)^{n}} d \mu(x)
\end{aligned}
$$

for all $n \geq 1$ that (A*) is satisfied. (We used the formula (G2) here). Further,

$$
\begin{aligned}
b_{r} & =\sum_{k \geq 0}(-1)^{k-r} a_{k} S_{1}(k, r) \\
& =\sum_{k \geq 0}(-1)^{k-r}\left[\int_{(0,2)} \frac{(x-1)^{k}}{k !} d \mu(x)\right] S_{1}(k, r) \\
& =\int_{(0,2)}\left[(-1)^{r} \sum_{k \geq 0} \frac{(1-x)^{k}}{k !} S_{1}(k, r)\right] d \mu(x) \\
& =\int_{(0,2)} \frac{(\log x)^{r}}{r !} d \mu(x)
\end{aligned}
$$

for all $r \geq 1$, so that $\left(\mathrm{B}^{*}\right)$ holds. (We used the formula (G1) here.)

Conversely, suppose $\mu$ is supported on $(0,2)$ and $\left(\mathrm{A}^{*}\right)$ and $\left(\mathrm{B}^{*}\right)$ hold. Our observations above show that

$$
\sum_{k \geq 0} \sum_{r \geq 0} A_{k} S_{1}(k, r) n^{r}<\infty
$$

for all $n \geq 0$. Clearly, $a_{2 k-1} \leq A_{2 k-1}$ for all $k \geq 1$ so that, to verify condition (A), we need only verify the finiteness of

$$
\sum_{k \geq 0} \sum_{r \geq 0} a_{2 k} S_{1}(2 k, r) n^{r} \quad(n \geq 0) .
$$

Note that $a_{2 k}=A_{2 k}+B_{2 k} \leq \frac{A_{2 k-1}}{2 k}+\frac{B_{2 k-1}}{2 k} \leq \frac{2 A_{2 k-1}}{2 k}$ for all $k \geq 1$. Using the recurrence relation (R1), we have

$$
\begin{aligned}
& a_{2 k} S_{1}(2 k, r) n^{r} \leq \frac{2 A_{2 k-1}}{2 k} S_{1}(2 k, r) n^{r} \\
& \quad \leq 2 A_{2 k-1} S_{1}(2 k-1, r) n^{r}+n A_{2 k-1} S_{1}(2 k-1, r-1) n^{r-1}
\end{aligned}
$$

for all $k$ and $n \geq 1$, and it is now clear that (A) holds. Further, (B*) yields the non-negativity of $b_{r}(r \geq 0)$ in view of our computations earlier so that (B) holds as well, and $\left\{\beta_{n}\right\}_{n \geq 0}$ falls in $\mathcal{A} M S^{*}$. 
Examples 2. (i) As noted in (ii) of Examples 1 above, the sequence $\left\{\beta_{n}=(1+\delta)^{n}\right\}_{n \geq 0}$, with $0 \leq \delta<1$, is a member of $\mathcal{A} M S^{*}$. The sequence $\left\{\beta_{n}\right\}_{n \geq 0}$ satisfies the hypotheses of Theorem 6 with the corresponding measure $\mu$ being the unit point mass at $(1+\delta)$. Clearly, the sequence $\left\{\beta_{n}\right\}_{n \geq 0}$ can be looked upon as $\left\{\theta_{n}(T)\right\}_{n \geq 0}$ corresponding to a weighted shift $T$ that is an alternatingly hyperexpansive subnormal operator.

(ii) The function $f(x)=\left(a^{x}+a^{-x}\right) / 2$, where $1<a<2$, defines a member of $\mathcal{A} M F^{*}$ such that the sequence $f(n)$ satisfies the hypotheses of Theorem 6; the corresponding weighted shift is alternatingly hyperexpansive and subnormal with the associated measure $\mu$ concentrated at the points $a$ and $1 / a$. On the other hand, the absolutely monotone moment sequence $\{\cosh (n)\}_{n \geq 0}$ does not satisfy the hypotheses of Theorem 6 .

(iii) In view of Theorems 2 and 3 , the sequence $\left\{\beta_{n}=f^{\prime}(n)\right\}_{n \geq 0}$ that is interpolated by the derivative $f^{\prime}(x)$ of the function $f(x)$ in (ii) above is a member of $\mathcal{A} M S^{*}$; however, the weighted shift $T$ with $\theta_{n}(T)=1+\beta_{n}$, though alternatingly hyperexpansive, is nonsubnormal.

Motivated by our observations so far, we raise the following questions.

Questions. If $T=\Gamma\left(\alpha_{n}\right)$ is such that $\left\{\theta_{n}(T)\right\}_{n \geq 0}$ is interpolated by a member of $\mathcal{A M F}^{*}$, does the $\operatorname{limit} \lim _{n \rightarrow \infty} \alpha_{n}$ exist? More generally, does the limit $\lim _{n \rightarrow \infty} \alpha_{n}$ exist if $\left\{\theta_{n}(T)\right\}_{n \geq 0}$ is interpolated by an absolutely monotone function on $\mathbb{R}_{+}$? In general, does the limit $\lim _{n \rightarrow \infty} \alpha_{n}$ exist for any alternatingly hyperexpansive weighted shift $T=\Gamma\left(\alpha_{n}\right)$ ?

\section{REFERENCES}

[1] A. Athavale, Alternatingly hyperexpansive operator tuples, Positivity 5 (2001), 259-273.

[2] C. Berg, J. P. R. Christensen and P. Ressel, Harmonic Analysis on Semigroups, Springer-Verlag, Berlin, 1984.

[3] J. B. Conway, The Theory of Subnormal Operator, Math. Surveys and Monographs, Vol. 36, Amer. Math. Soc., Providence, Rhode Island, 1991.

[4] M. R. Embry, A generalization of the Halmos-Bram criterion for subnormality, Acta Sci. Math. (Szeged) 31 (1973), 61-64.

[5] R. L. Graham, D. E. Knuth and O. Patashnik, Concrete Mathematics, Addison-Wesley, New York, 1994. 
[6] F. B. Hildebrandt, Introduction to Numerical Analysis, McGraw-Hill, New York, 1974.

[7] Zenon J. Jablonski, Complete hyperexpansivity, subnormality and inverted boundedness conditions, Int. Eq. Operator Th. 44 (2002), 316-336.

[8] W. Rudin, Real and Complex Analysis, McGraw-Hill, New York, 1986.

[9] V. M. Sholapurkar and A. Athavale, Completely and alternatingly hyperexpansive operators, J. Operator Theory 43 (2000), 43-68.

[10] A. Shields, Weighted shift operators and analytic function theory, Topics in Operator Theory, Math. Surveys 13, Amer. Math. Soc., Providence, R.I. (1974), 49-128.

[11] D. Widder, The Laplace Transform, Princeton University Press, London, 1946.

Ameer Athavale,

Department of Mathematics,

Indian Institute of Technology Bombay,

Mumbai 40007,

India

athavale@math.iitb.ac.in

\author{
Abhijit Ranjekar, \\ Department of Mathematics, \\ University of Pune, \\ Pune 411007, \\ India \\ abhijit@math.unipune.ernet.in
}

V. M. Sholapurkar,

S. P. College,

Pune 411030,

India

v_sholapurkar@vsnl.net 